\title{
Decaimento das Soluções para uma Mistura de Sólidos Timoshenko Termoelástico
}

\author{
Félix Pedro Quispe Gómez \\ Departamento Académico de Matemática, DAMAT - UTFPR, \\ 80230-901, Curitiba, PR \\ E-mail: felixgomez@utfpr.edu.br, felix12gomez@gmail.com
}

\section{$\underline{\text { RESUMO }}$}

Abordaremos o caso de uma viga de Timoshenko unidimensional composta por uma mistura de dois meios contínuos interagindo e que ocupam o intervalo $] 0, L[$. As variáveis dependentes que formam as equações de Timoshenko serão representadas por $\varphi$ e $\psi$. Quando se estuda a mistura de dois meios interagindo caracterizamos o deslocamento das partículas utilizando a seguinte notação $\varphi^{1}=\varphi^{1}(x, t), \psi^{1}=\psi^{1}(x, t)$ e $\varphi^{2}=\varphi^{2}(y, t), \psi^{2}=\psi^{2}(y, t)$ onde $\left.x, y \in\right] 0, L[$. Supondo que as partículas em nosso estudo ocupam a mesma posição no tempo $t=0$ de modo que $x=y$. Ver os trabalhos de [1], [3] e [7].

Denotamos por $\theta=\theta(x, t)$ a temperatura do material no ponto $x$ e no instante $t$.

Sejam as equações dos tensores de estresse

$$
\begin{aligned}
& S^{1}=k_{11}\left(\partial_{x} \varphi^{1}+\psi^{1}\right)+k_{12}\left(\partial_{x} \varphi^{2}+\psi^{2}\right) \\
& S^{2}=k_{12}\left(\partial_{x} \varphi^{1}+\psi^{1}\right)+k_{22}\left(\partial_{x} \varphi^{2}+\psi^{2}\right)
\end{aligned}
$$

e os correspondentes momentos para cada componente

$$
\begin{aligned}
& M^{1}=b_{11} \partial_{x} \psi^{1}+b_{12} \partial_{x} \psi^{2}-\beta_{1} \theta \\
& M^{2}=b_{12} \partial_{x} \psi^{1}+b_{22} \partial_{x} \psi^{2}-\beta_{2} \theta
\end{aligned}
$$

Portanto as equações do movimento e a equação da energia podem ser escritas em termos das funções $\varphi, \psi$ e $\theta$

$$
\begin{aligned}
\rho_{11} \partial_{t}^{2} \varphi^{1}+\alpha_{1}\left(\varphi^{1}-\varphi^{2}\right)-\partial_{x} S^{1} & =0 \\
\rho_{12} \partial_{t}^{2} \varphi^{2}-\alpha_{1}\left(\varphi^{1}-\varphi^{2}\right)-\partial_{x} S^{2} & =0 \\
\rho_{21} \partial_{t}^{2} \psi^{1}-\partial_{x} M^{1}+\alpha_{2}\left(\psi^{1}-\psi^{2}\right)+S^{1} & =0 \\
\rho_{22} \partial_{t}^{2} \psi^{2}-\partial_{x} M^{2}-\alpha_{2}\left(\psi^{1}-\psi^{2}\right)+S^{2} & =0 \\
c \partial_{t} \theta-k \partial_{x}^{2} \theta+\beta_{1} \partial_{x t}^{2} \psi^{1}+\beta_{2} \partial_{x t}^{2} \psi^{2} & =0
\end{aligned}
$$

A seguir escrevemos as equações acima na forma de funções vetoriais, para isso consideramos as seguintes matrizes

$$
\begin{aligned}
\rho^{i} & =\left[\begin{array}{cc}
\rho_{i 1} & 0 \\
0 & \rho_{i 2}
\end{array}\right] & \varphi=\left[\begin{array}{l}
\varphi^{1} \\
\varphi^{2}
\end{array}\right] & \psi=\left[\begin{array}{l}
\psi^{1} \\
\psi^{2}
\end{array}\right] \\
S & =\left[\begin{array}{l}
S^{1} \\
S^{2}
\end{array}\right] & M & =\left[\begin{array}{l}
M^{1} \\
M^{2}
\end{array}\right] \\
B & =\left[\begin{array}{ll}
b_{11} & b_{12} \\
b_{12} & b_{22}
\end{array}\right] & K=\left[\begin{array}{ll}
k_{11} & k_{12} \\
k_{12} & k_{22}
\end{array}\right] & B_{i}=\alpha_{i}\left[\begin{array}{cc}
1 & -1 \\
-1 & 1
\end{array}\right]
\end{aligned}
$$


Assim sendo, temos o sistema vetorial para as equações de movimento e energia

$$
\begin{aligned}
\rho^{1} \partial_{t}^{2} \varphi-\partial_{x} S+B_{1} \varphi & =0 \\
\rho^{2} \partial_{t}^{2} \psi-\partial_{x} M+B_{2} \psi+S & =0 \\
c \partial_{t} \theta-k \partial_{x}^{2} \theta+\beta \cdot \partial_{x t}^{2} \psi & =0
\end{aligned}
$$

Substituindo as derivadas das expressões $S$ e $M$ obtemos

$$
\begin{aligned}
\rho^{1} \partial_{t}^{2} \varphi-K \partial_{x}\left(\partial_{x} \varphi+\psi\right)+B_{1} \varphi & =0 \\
\rho^{2} \partial_{t}^{2} \psi-B \partial_{x}^{2} \psi+K\left(\partial_{x} \varphi+\psi\right)+B_{2} \psi+\beta \partial_{x} \theta & =0 \\
c \partial_{t} \theta-k \partial_{x}^{2} \theta+\beta \cdot \partial_{x t}^{2} \psi & =0
\end{aligned}
$$

Para trabalhos bem próximos ver [9], [11] e [12].

O objetivo principal será obter condições sobre os coeficientes para garantir que o eixo imaginário esteja contido no conjunto resolvente. Logo, identificar as condições necessárias e suficientes para obter o decaimento exponencial das soluções. A técnica a ser utilizada é a de semigrupos e técnicas multiplicativas.

Palavras-chave: Misturas Termoelásticas, Decaimento exponencial, Viga de Timoshenko, Sistemas Acoplados.

\section{Referências}

[1] R. J. Atkin and R. E. Craine; Continuum theories of mixtures: basic theory and historical development, Quat. J. Mech. Appl. Math. 29, 209-243, (1976).

[2] A. Bedford and D. S. Drumheller; Theory of immiscible and strucured mixtures, Internat. J. Engrg Sci., 21, 863-960, (1983).

[3] A. Bedford and M. Stern; A multi-continuum theory for composite elastic materials, Acta Mechanica, 14, 85-102, (1972).

[4] A. E. Green and P. M. Naghdi; A dynamical theory of interacting continua, Internat. J. Engrg Sci., 3, 231-241, (1965).

[5] A. E. Green and P. M. Naghdi; A note on mixtures, Internat. J. Engrg Sci., 6, 631-635, (1968).

[6] A.E. Green and T.R. Steel; Constitutive equations for interacting continua. Internat. J. Engrg Sci., 4, 483-500, (1966).

[7] D. Ieşan; On the theory of mixtures of thermoelastic solids. J. Thermal Stresses, 14, 389-408, (1991).

[8] D. Ieşan and R. Quintanilla; Existence and continuous dependence results in the theory of interacting continua. J. Elasticity, 35, 85-98, (1994).

[9] F. Martínez and R. Quintanilla; Some qualitative results for the linear theory of binary mixtures of thermoelastic solids. Collect. Math., 46, 263-277, (1995).

[10] K. R. Rajagopal and L. Tao; Mechanics of Mixtures, World Scientific, Singapore, 1995.

[11] T.R. Steel; Applications of a theory of interacting continua, Quarterly Journal of Mechanics and Applied Mathematics, 20, 57-72, (1967).

[12] Zenhg, S. On the theory of mixtures of thermoelastic solids, J. Thermal Stresses, 14, 389-408, (1991) 